\title{
STARSPOT ACTIVITIES AS AN INTERPRETATION FOR THE \\ LIGHT CURVE CHANGES OF THE CLOSE BINARY
}

\author{
STAR XY UMa
}

\author{
E. H. GEYER \\ Observatorium Hoher List - Astronomische Institute der Universität Bonn, F.R.G.
}

\begin{abstract}
The large light curve changes of XY UMa, observed in the last $20 \mathrm{yr}$, are interpreted as stellar starspot activity of the primary component of this binary.
\end{abstract}

The 0.4799-day eclipsing binary XY UMa is a unique star as far as its photometric hehaviour is concerned. Shortly after its variability was found by Strohmeier, and its period determined by Kippenhahn (Geyer et al., 1955) I started photoelectric observations, mainly in $B$ and $V$, of this object and sporadically continued them up to 1975 . Eight fairly complete light curves have been obtained in the years 1955, 1956, 1957, 1958, 1959, 1961,1968 and 1975 . They show not only large variations between consecutive observing nights, but also that the character of the light curves underwent severe changes within this 20-yr period of photoelectric observation.

Some 30 grating spectrograms covering all phases $\left(136 \AA \mathrm{mm}^{-1}\right.$, spectral range from 6700 to $3700 \AA$ ) always show a G2V-type spectrum with no trace of emission lines, excluding therefore the existence of detectable circumstellar matter.

The observational facts can be summarized as follows:

(1) The revolution period of 0.478995 days was constant during $20 \mathrm{yr}$ (15250 epochs!).

(2) The average brightness of the binary system changed between 1955 and 1975 in a sinusoidal manner by $0.18 \mathrm{mag}$ in $V$ and $0.20 \mathrm{mag}$ in $B$, indicating a periodic variation of about 28 to $30 \mathrm{yr}$. The lowest system brightness was observed in 1961, and the highest in 1975.

(3) Rather symmetrical light curves of $\beta$-Lyrae type, showing large scatter of the observations in maximum light which exceeds the photometric accuracy by at least five times, occurred in 1958, 1959 and 1968. Colour index at primary minimum is redder than at secondary minimum. The latter appears to be an occultation, so that the hotter component is also the larger one. The maxima differ only slightly, maximum I being brighter in 1958/59 than maximum II, and the reverse in 1968.

(4) An asymmetric light curve appeared in 1961. A deep, more or less symmetric primary minimum was followed by a curved maximum at phase 0.2 . Large scatter at this point and the following declining branch towards secondary minimum was observed. In the phase interval 0.6 to 0.9 the light curve exhibited a plateau which was only insignificantly brighter than the secondary minimum, and the noise was reduced.

(5) The light curve observed in 1975 is in many respects a mirror image of the 1961 curve, and resembles that of a dwarf-nova eclipsing star. The main changes from the 1961 observations are that the primary minimum is shallower and also wider, and the scatter over nearly all the light curve is of the order of the observational errors. The system brightness which was lowest in 1961 now has reached the highest observed value.

For the interpretation of these phenomena I propose the following model for XY UMa. The two components of this binary are dwarf stars, the primary being of spectral type G2, 


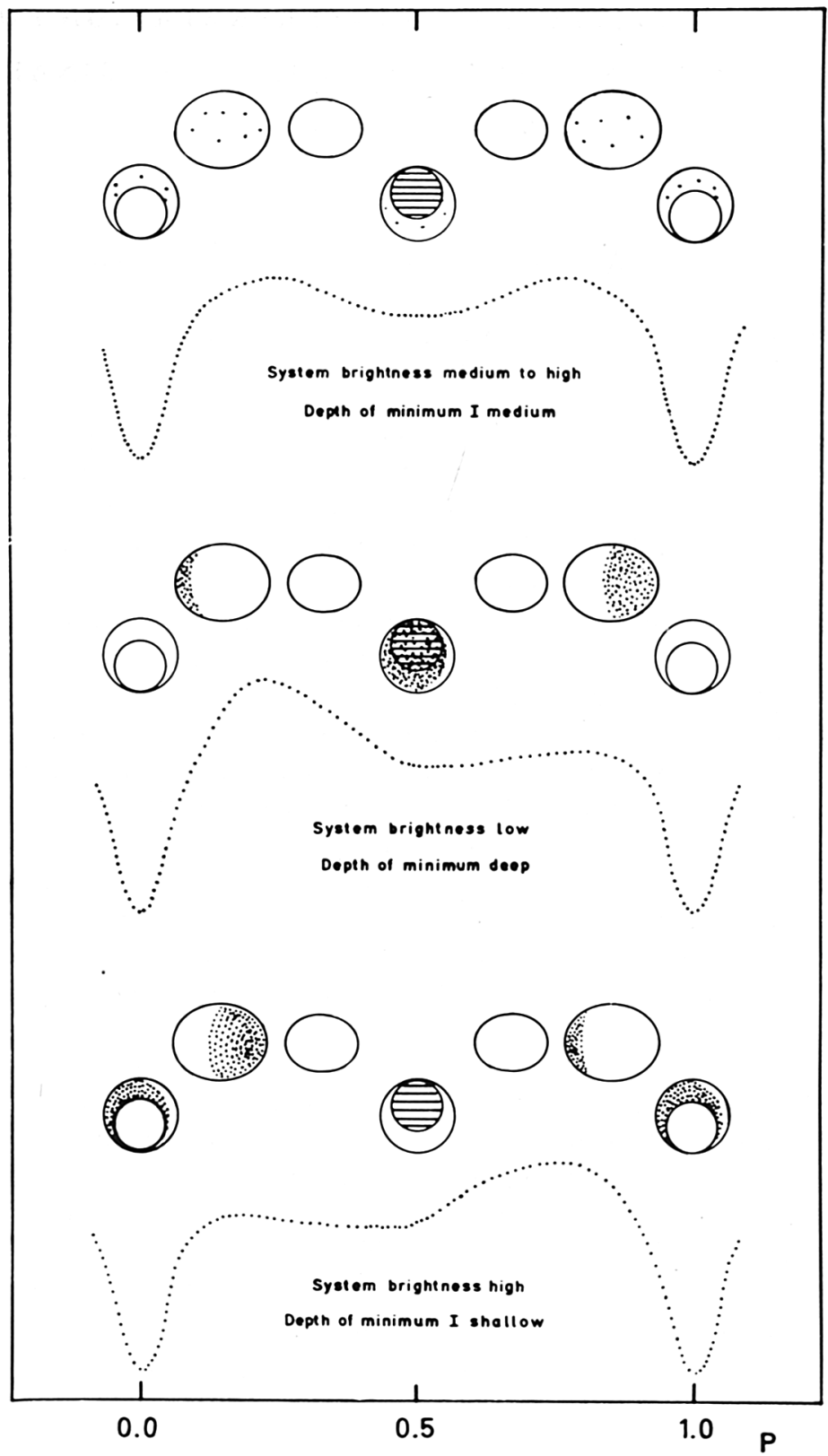

Fig. 1. Schematic diagram for the interpretation of the light curve changes of XY UMa by stellar spot areas. 
the secondary perhaps of type K5. Both stars are well below their Roche limit configuration. The primary component shows a larger starspot activity, the cycle of which is about $15 \mathrm{yr}$ (or double that value). In years of lower activity or when the dark spots appear in a more uniform spread in longitude over the stellar sphere, the more symmetrical light curves appear, and the system brightness may reach the highest value for the observer (Figure 1, upper curve). When on the other hand the stellar spot activity is higher and (or) more concentrated to a narrower longitude interval of the stellar sphere, asymmetric light curves with the described peculiarities occur. In 1961 the spotted area of the G2 component of XY UMa was on the far side of the observer during primary minimum. Thus a larger light loss was caused by the transit eclipse of the smaller component. Under these conditions the observer, outside of the primary eclipse, always sees parts of the spotted area, and this phenomenon can be well observed shortly after the secondary component is occulted. This causes the plateau shaped part of the light curve, and at the same time the system brightness is at its lowest value (Figure 1, middle curve). On the other hand if the stellar activity zone is more or less directed towards the observer during primary eclipse as it was the case in 1975, the light loss is less, and the minimum therefore shallow. The whole dark spotted area is presented to the observer shortly after this phase, and disappears slightly before phase 0.5 . The flat topped shape now occurs in that part of the light curve, and at the same time the system brightness is high (Figure 1, lower curve).

To explain the irregularities in the light curves of W UMa systems Binnendijk (1970) proposed the existence of subluminous areas on the surfaces of their primary components. Only recently theoretical arguments for the forming of magnetic starspots on the primary components of such binary systems were given by Mullan (1975).

Since stellar spot activity may also be connected with a kind of flare activity I would like to make a prediction about XY UMa: whenever its spotted area is pointing towards the observer over most phases, soft X-ray emission may be switched on and therefore observable.

\section{References}

Binnendijk, L.: 1970, in A. Beer (ed.), Vistas in Astronomy, Vol. 12, Pergamon Press, p. 217.

Geyer, E. H., Kippenhahn, R., and Strohmeier, W.: 1955, Kleine Veroeffentl. Remeis Sternwarte Bamberg, No. 9.

Mullan, D. J.: 1975, Astrophys. J. 198, 563.

\section{DISCUSSION}

Hilditch: I would just like to comment that such light curve variations have been observed in another short period G-type system, namely SV Cam. I have unpublished observations obtained in 1970 which show a 'hump' around phase 0.20 . More recent data in 1974 indicate that the system has returned to a normal situation with a symmetric light curve.

Geyer: Thank you for drawing my attention to this object!

Hall: In case you are not entirely familiar with the RSCVn binaries, let me point out that all of the remarkable properties you observe in XY UMa are observed in the RSCVn binaries.

Leung: Your result is a good example of a variable light curve. Dr Hilditch may agree with me that XY UMa is very similar to the system of SV Cam. I would like to draw your attention to the system of VW Cep, which also has similar ions in its light curve.

Geyer: Many W UMa systems like VW Cep show larger light curve changes of similar character to those I showed for XY UMa. Therefore the assumption of star-spot activity seems to be quite realistic. 
De Loore: Are there traces of magnetic fields in these systems? Does Mullan report on the magnetic field of the secondary in his list?

Geyer: The system is too faint to look for magnetic fields. The paper of Mullan which appeared in June 15 edition of Astrophysical Journal demands magnetic starspot activity for the primary component of W UMa binaries.

Hall: Let me clarify the situation with the Mullen reference. Mullen's first paper primarily concerned the flare star but indicated that the results could probably be useful in understanding related binaries displaying similar spot phenomena. In a lat ir paper he did indeed extend the application of his work to certain W UMa binaries which seem to show spotted or subluminous regions.

At IAU Colloquium No. 29 in Budapest I will review the RSCVn binaries, and binaries displaying similar properties. These related groups include binaries like CG Cyg with orbital periods less than 1 day, those like HK Lac with periods greater than 2 weeks, flare star binaries like YY Gem, certain wtype W UMa binaries like VW Cep, and V471 Tauri. Thus XY UMa would belong to the short period group which contains CG Cyg, RT And, SV Cam, WY Cnc, UV Psc, and ER Vul but would not be a bona fide RSCVn binary as I define them in this review paper. 